\title{
Ueber eine Eigenschaft der Kugelfunctionen.
}

\author{
(Von Herrn A. Clebsch zu Carlsruhe.)
}

W enn man eine Function dreier rechtwinkligen Coordinaten nach Kugelfunctionen entwickelt, so gelangt man zu einer Reihe, deren Glieder die bekannten Functionen zweier Winkel sind, während in ihren Coefficienten nur der Radius Vector noch auftritt. Jene Functionen zweier Winkel haben aber die Eigenschaft, dass sie, mit einer passenden Potenz des Radius multiplicirt, in ganze homogene Functionen der Coordinaten übergehen; man kann daher die Entwicklung nach homogenen Functionen der Coordinaten ausführen, deren Coefficienten dann nur noch Functionen des Radius Vector sind. Diese homogenen Functionen, welche in einigen Aufgaben der mathematischen Physik ihrer Symmetrie wegen mit Vortheil angewandt werden können, sind dann dadurch definirt, dass sie neben der Bedingung der Homogeneität der Gleichung

$$
\text { (1.) } \Delta^{2} \Omega=0
$$

(nach Lamés Bezeichnung) genügen. Eine grosse Zahl von Eigenschaften dieser Functionen erhält man direct aus den bekannten Eigenschaften der Kugelfunctionen; eine etwas ferner liegende Eigenschaft bildet den Gegenstand dieses Aufsatzes.

Zuvörderst kann man leicht die allgemeinste Gestalt der Function $n^{\text {ter }}$ Ordnung angeben, welche der Gleichung (1.) genügt. Es sei zu diesem Zweck

$$
\text { (2.) } \Omega=V+r^{2} V_{1}+r^{4} V_{2} \ldots,
$$

wo $r$ den Radius Vector bedeutet, und wo $V, V_{1}, V_{2} \ldots$ homogene Functionen respective der $n^{\text {ten }}, n-2^{\text {ten }}, n-4^{\text {ten }} \ldots$ Ordnung bedeuten. Die Form (2.) kann offenbar auf unendlich viele Arten gewählt werden; da $V$ noch beliebig gelassen ist, kann man über $V_{1}, V_{2} \ldots$ jedenfalls nach Willkür verfügen. Inzwischen ist

$$
\begin{aligned}
\Delta^{2}\left(r^{2 k} V_{k}\right) & =r^{2 k} \Delta^{2} V_{k}+4 k\left(x \frac{\partial V_{k}}{\partial x}+y \frac{\partial V_{k}}{\partial y}+z \frac{\partial V_{k}}{\partial z}\right) r^{2 k-2}+2 k(2 k+1) r^{2 k-2} V_{k} \\
& =r^{2 k} \Delta^{2} V_{k}+2 k(2 n-2 k+1) V_{k} r^{2 k-2} .
\end{aligned}
$$

Die Gleichung (1.) geht daher durch Substitution von (2.) über in:

$$
\begin{aligned}
0= & \Delta^{2} V+r^{2} \Delta^{2} V_{1}+r^{4} \Delta^{2} V_{2} \ldots \\
& +2(2 n-1) V_{1}+4(2 n-3) r^{2} V_{2}+6(2 n-5) r^{4} V_{3} \ldots
\end{aligned}
$$


In dieser Gleichung kann man nun das letzte $V$ so bestimmen, dass der Coefficient der höchsten Potenz von $r$ verschwindet; sodann das vorletzte $V$ so, dass auch die zweithöchste Potenz von $r$ verschwindet, u. s. w. Dann bleibt schliesslich eine Gleichung, welche $V_{1}$ durch $V$ ausdrückt; und indem dies letztere völlig unbestimmt bleibt, hat man die Beziehungen:

$$
\begin{aligned}
& V_{1}=-\frac{\Delta^{2} V}{2.2 n-1}, \\
& V_{2}=-\frac{\Delta^{2} V_{1}}{4.2 n-3}, \\
& V_{3}=-\frac{\Delta^{2} V_{2}}{6.2 n-5},
\end{aligned}
$$

oder, wenn man durch $\Delta^{4}, \Delta^{6} \ldots$ die doppelte, dreifache etc. Wiederholung der Operation $\Delta^{2}$ andeutet:

$$
\begin{aligned}
& V_{1}=-\frac{\Delta^{2} V}{2.2 n-1}, \\
& V_{2}=+\frac{\Delta^{4} V}{2.4 .2 n-1.2 n-3}, \\
& V_{3}=-\frac{\Delta^{6} V}{2.4 .6 .2 n-1.2 n-3.2 n-5},
\end{aligned}
$$

Man hat also sofort den Satz:

Die allgemeinste ganze Function $n^{\text {ter }}$ Ordnung, welche der Gleichung $\Delta^{2} \Omega=0$ genügt, ist

$$
\text { (3.) } \Omega=V-\frac{r^{2} \cdot \Delta^{2} V}{2.2 n-1}+\frac{r^{4} \cdot \Delta^{4} V}{2.4 .2 n-1.2 n-3}-\cdots,
$$

wo $V$ eine ganz beliebige homogene Function $n^{\text {ter }}$ Ordnung bedeutet $\left.{ }^{*}\right)$.

Setzt man hier z. B. $V=x^{n}$, so erhält man die der Kugelfunction $\boldsymbol{P}_{n}$ entsprechende homogene Function; setzt man $V=\left(x x_{0}+y y_{0}+z z_{0}\right)^{n}$, so ergeben

*) Man kann den obigen Satz noch dahin ohne Mühe erweitern, dass die allgemeinste Function $n^{\text {ter }}$ Ordnung, welche der Gleichung

$$
\Delta^{2} \Omega=\vartheta
$$

genügt, (wo $\vartheta$ eine ganze homogene Function $(n-2)^{\text {ter }}$ Ordnung bedeutet) folgende Gestalt annimmt:

$$
\begin{aligned}
\Omega=\frac{r^{2} \vartheta}{2.2 n-1}-\frac{r^{4} \Delta^{2} \vartheta}{2.4 .2 n-1.2 n-3}+ & \frac{r^{6} \Delta^{4} \vartheta}{2.4 .6 .2 n-1.2 n-3.2 n-5}-\cdots \\
& +V-\frac{r^{2} \Delta^{2} V}{2.2 n-1}+\frac{r^{4} \Delta^{4} V}{2.4 .2 n-1.2 n-3}-+\cdots
\end{aligned}
$$


sich diejenigen Ausdrücke, welche bei der Entwickelung der Quadratwurzel der reciproken Entfernung zweier Punkte auftreten. Setzt man aber in (3.) $V=r^{2} . W$, wo $W$ von $\operatorname{der}(n-2)^{\text {ten }}$ Ordnung ist, so entsteht immer identisch Null. Denn man erhält offenbar immer

$$
\Delta^{2 k}\left(r^{2} W\right)=r^{2} \Delta^{2 k} W+\alpha_{k} \Delta^{2 k-2} W,
$$

und indem man auf diese Gleichung die Operation $\Delta^{2}$ anwendet:

$$
\Delta^{2 k+2}\left(r^{2} W\right)=r^{2} \Delta^{2 k+2} W+\left(\alpha_{k}+4 n-8 k-2\right) \Delta^{2 k} W .
$$

Es ist daher

$$
\alpha_{k+1}=\alpha_{k}+4 n-8 k-2 ;
$$

und wenn man bemerkt, dass $\alpha_{0}=0$, so ergiebt sich sofort:

$$
\alpha_{k+1}=2(k+1)(2 n-2 k-1), \quad \alpha_{k}=2 k(2 n-2 k+1) .
$$

Man hat sonach

$$
\Delta^{2 k}\left(r^{2} W\right)=r^{2} \Delta^{2 k} W+2 k(2 n-2 k+1) \Delta^{2 k-2} W ;
$$

und wenn man also $V=r^{2} W$ setzt:

$$
\Omega=r^{2} W-\frac{r^{4} \Delta^{2} W+2(2 n-1) r^{2} W}{2.2 n-1}+\frac{r^{6} \Delta^{4} W+4(2 n-3) r^{4} \Delta^{2} W}{2.4 .2 n-1.2 n-3}-\cdots
$$

was offenbar identisch verschwindet.

Hierdurch erklärt es sich, dass die in der allgemeinen Form von $V$ enthaltenen $\frac{n+1 . n+2}{1.2}$ Constanten nur die Stelle von $2 n+1$ Constanten vertreten, wie dies die bekannte Thatsache fordert, dass die allgemeinste Kugelfunction $n^{\text {ter }}$ Ordnung nur die letztere Zahl willkürlicher Constanten enthält. Denn dem Obigen zufolge kann man in (3.) ohne die Allgemeinheit zu beeinträchtigen $V-r^{2} W$ an die Stelle von $V$ treten lassen, und man kann dann die $\frac{n . n+1}{1.2}$ Coefficienten von $W$ so bestimmen, dass ebensoviel Coefficienten in $V$ bestimmte Werthe annehmen.

Ich werde nun zeigen, dass man immer für $\boldsymbol{V}$ das Product von $n$ linearen Factoren setzen darf, und werde zugleich zeigen, wie man dieselben findet, indem man $W$ so bestimmt, dass $V-r^{2} W$ in lineare Factoren sich auflöst.

Denken wir uns unter $x, y, z$ die homogenen Coordinaten eines Punktes in der Ebene. Dann ist

$$
\boldsymbol{V}=\mathbf{0}
$$

die Gleichung einer Curve $\boldsymbol{n}^{\text {ter }}$ Ordnung; und dieselbe wird von dem Kegelschnitt

$$
r^{2}=x^{2}+y^{2}+z^{2}=0
$$

Journal für Mathematik Bd. LX. Heft 4. 
in 2n Punkten geschnitten. Verbindet man diese Punkte paarweise durch $n$ gerade Linien, so dass durch jeden Punkt dabei eine Linie geht (was auf 1.3.5...2n-1 Arten geschehen kann) und sind

$$
\alpha_{1}=0, \quad \alpha_{2}=0, \ldots \alpha_{n}=0
$$

die Gleichungen solcher $n$ Geraden; so kann man bekanntlich, da weder $\boldsymbol{V}=\mathbf{0}$ noch diese Geraden von $r=0$ in anderweitigen Punkten geschnitten werden, die absoluten Werthe der Coefficienten in den $\alpha$ so bestimmen, dass identisch

$$
V=\alpha_{1} \alpha_{2} \ldots \alpha_{n}+r^{2} W
$$

wo $W$ eine homogene Function $(n-2)^{\text {ter }}$ Ordnung bedeutet.

Hierdurch ist die oben aufgestellte Frage erledigt, und es fragt sich nur, wie man für eine gegebene Function $\boldsymbol{\Omega}$ dieses Product von Geraden oder die erwähnten $2 n$ Schnittpunkte bestimmen könne, selbst wenn $\Omega$ nicht in der Form (3.) gegeben vorliegt. Nun bemerkt man aus (3.), dass die Schnittpunkte der Curven

$$
V=0, \quad x^{2}+y^{2}+z^{2}=0
$$

mit den Schnittpunkten von

$$
\text { (4.) } \Omega=0, \quad x^{2}+y^{2}+z^{2}=0
$$

zusammenfallen. Man hat also nur die letzteren aufzusuchen; eine Aufgabe, welche durch die eigenthümliche Natur der Function $\Omega$ wesentlich erleichtert wird.

Die Gleichung $2 n^{\text {ten }}$ Grades, auf welche die vorliegende Aufgabe führt, kann man in der Weise aufstellen, dass man das Product aller Gleichungen der $2 n$ Schnittpunkte aufsucht. Dieser Ausdruck muss dann, was mit Hülfe einer Gleichung $2 n^{\text {ten }}$ Grades sofort geschehen kann, in seine $2 n$ Factoren aufgelöst werden, und es ist leicht dann die Gleichungen $\alpha_{1}=0, \alpha_{2}=0, \ldots \alpha_{n}=0$ zu bilden. Um aber jenes Product zu finden, verbindet man die Gleichungen (4.) mit der Gleichung

$$
\text { (5.) } \quad u x+v y+w z=0
$$

eines Schnittpunktes, und eliminirt $x, y, z$. Eine solche Elimination, bei welcher eine Gleichung vom $1^{\text {ten }}$, die zweite vom $2^{\text {ten }}$, die dritte vom $n^{\text {ten }}$ Grade ist, habe ich im $58^{\text {ten }}$ Bd. d. J. p. 273 allgemein ausgeführt; und zwar wird das Resultat nach den dort gegebenen Regeln:

$$
\text { (6.) } \quad R=0=\frac{1}{2}\left[\left\{C+\sqrt{C^{2}-A B}\right\}^{n}+\left\{C-\sqrt{C^{2}-A B}\right\}^{n}\right] \text {, }
$$

wo, nach den hier eintretenden Werthen der Coefficienten der Gleichung zweiten Grades: 
(7.) $\quad A=\left|\begin{array}{lllll}1 & 0 & 0 & u & a \\ 0 & 1 & 0 & v & b \\ 0 & 0 & 1 & w & c \\ u & v & w & 0 & 0 \\ a & b & c & 0 & 0\end{array}\right|, \quad B=\left|\begin{array}{ccccc}1 & 0 & 0 & u & a^{\prime} \\ 0 & 1 & 0 & v & b^{\prime} \\ 0 & 0 & 1 & w & c^{\prime} \\ u & v & w & 0 & 0 \\ a^{\prime} & b^{\prime} & c^{\prime} & 0 & 0\end{array}\right|, \quad C=\left|\begin{array}{ccccc}1 & 0 & 0 & u & a^{\prime} \\ 0 & 1 & 0 & v & b^{\prime} \\ 0 & 0 & 1 & w & c^{\prime} \\ u & 0 & w & 0 & 0 \\ a & b & c & 0 & 0\end{array}\right|$,

wo endlich $a, b, c, a^{\prime}, b^{\prime}, c^{\prime}$ symbolische Coefficienten sind, welche durch die symbolischen Gleichungen

definirt werden.

$$
\text { (8.) } \quad \Omega=(a x+b y+c z)^{n}=\left(a^{\prime} x+b^{\prime} y+c^{\prime} z\right)^{n}
$$

Inzwischen ist nach (7.):

(9.) $\left\{\begin{array}{l}A=\left(u^{2}+v^{2}+w^{2}\right)\left(a^{2}+b^{2}+c^{2}\right)-(u a+v b+v c)^{2} ; \text { und ebenso } \\ B=\left(u^{2}+v^{2}+w^{2}\right)\left(a^{\prime 2}+b^{\prime 2}+c^{\prime 2}\right)-\left(u a^{\prime}+v b^{\prime}+w c^{\prime}\right)^{2}, \\ C=\left(u^{2}+v^{2}+w^{2}\right)\left(a a^{\prime}+b b^{\prime}+c c^{\prime}\right)-(u a+v b+w c)\left(u a^{\prime}+v b^{\prime}+v c^{\prime}\right) .\end{array}\right.$

$$
\boldsymbol{A}=(\boldsymbol{v c}-\boldsymbol{w b})^{2}+(\boldsymbol{w a}-u \boldsymbol{c})^{2}+(\boldsymbol{u b}-\boldsymbol{v a})^{2}, \quad \text { oder }
$$

Diese Gleichungen vereinfachen sich wesentlich durch die Bemerkung, dass nach (8.) die symbolische Gleichung

$$
\begin{aligned}
0=\dot{\Delta}^{2} \Omega & =(a x+b y+c z)^{n-2} \cdot n \cdot n-1 \cdot\left(a^{2}+b^{2}+c^{2}\right) \\
& =\left(a^{\prime} x+b^{\prime} x+c^{\prime} z\right)^{n-2} \cdot n \cdot n-1 \cdot\left(a^{\prime 2}+b^{\prime 2}+c^{\prime 2}\right)
\end{aligned}
$$

stattfindet. Dieser zufolge darf man also wegen der besonderen Natur der Function $\Omega$ immer

$$
\text { (10.) } a^{2}+b^{2}+c^{2}=a^{\prime 2}+b^{\prime 2}+c^{\prime 2}=0
$$

setzen. Daher reduciren $A, B$ sich auf ihre letzten Glieder; und die Gleichung (6.) nimmt folgende Gestalt an:

wo

$$
\text { (11.) } \quad R=0=\frac{1}{2}\left(s-t+\sqrt{s^{2}-2 t}\right)^{n}+\frac{1}{2}\left(s-t-\sqrt{s^{2}-2 t}\right)^{n} \text {, }
$$

(12.) $s=\left(u^{2}+v^{2}+w^{2}\right)\left(a a^{\prime}+b b^{\prime}+c c^{\prime}\right), \quad t=(u a+v b+w c)\left(u a^{\prime}+v b^{\prime}+w c^{\prime}\right)$ gesetzt ist.

Führt man den Ausdruck (11.) aus, so erhält man:

$$
\text { (13.) } \quad R=0=\alpha t^{n}+\alpha_{1} t^{n-1} s+\alpha_{2} t^{n-2} s^{2}+\cdots
$$

wo $\alpha, \alpha_{1}, \ldots$ numerische Coefficienten sind, welche durch die identische Gleichung

(14.) $\alpha \varepsilon^{n}+\alpha_{1} \varepsilon^{n-1}+\alpha_{2} \varepsilon^{n-2}+\cdots=\frac{1}{2}\left\{(1-\varepsilon+\sqrt{1-2 \varepsilon})^{n}+(1-\varepsilon-\sqrt{1-2 \varepsilon})^{n}\right\}$

bestimmt sind. Man bemerkt aber sofort, dass mit Rücksicht auf die Glei44 * 
chungen (10.), und wenn man die Operation $\Delta^{2}$ jetzt mit den Veränderlichen $u, v, w$ statt mit $x, y, z$ ausführt:

$$
\Delta^{2}\left(t^{n-k}\right)=2(n-k)^{2} \cdot t^{n-k-1}\left(a a^{\prime}+b b^{\prime}+c c^{\prime}\right),
$$

daher überhaupt

$$
A^{2 k}\left(t^{n}\right)=2^{k} \cdot n^{2} \cdot(n-1)^{2} \ldots(n-k+1)^{2} \cdot t^{n-k} \cdot\left(a a^{\prime}+b b^{\prime}+c c^{\prime}\right)^{k},
$$

oder, wenn man der Kürze wegen

$$
\rho^{2}=u^{2}+v^{2}+w^{2}, \quad a a^{\prime}+b b^{\prime}+c c^{\prime}=\beta
$$

setzt:

$$
t^{n-k} \cdot \beta^{k}=\rho^{2 k} \cdot \frac{\Delta^{2 k}\left(t^{n}\right)}{2^{k} \cdot(n \cdot n-1 \ldots n-k+1)^{2}} .
$$

Bezeichnet man nun durch $U$ diejenige Function, in welche $\Omega$ übergeht, wenn man darin $u, v, w$ für $x, y, z$ substituirt, so hat man offenbar auch

$$
t^{n}=U^{2}
$$

und somit geht die Gleichung (13.) über in die merkwürdige Form:

$$
0=\alpha U^{2}+\alpha_{1} \frac{\varrho^{2} \Delta^{2}\left(U^{2}\right)}{2 . n^{2}}+\alpha_{2} \frac{\varrho^{4} \Delta^{4}\left(U^{2}\right)}{2^{2} \cdot(n \cdot n-1)^{2}}+\cdots,
$$

aus welcher die symbolischen Coefficienten sämmtlich verschwunden sind.

Sonach bleibt nur übrig, die Coefficienten $\alpha$ der Gleichung (14.) gemäss zu bestimmen, welche auch die Form annimmt:

$$
\alpha \varepsilon^{n}+\alpha_{1} \varepsilon^{n-1}+\alpha_{2} \varepsilon^{n-2}+\cdots=\frac{1}{2^{n+1}}\left\{(1+\sqrt{1-2 \varepsilon})^{2 n}+(1-\sqrt{1-2 \varepsilon})^{2 n}\right\} .
$$

Nun sind offenbar

$$
x=1+\sqrt{1-2 \varepsilon} \text { und } y=1-\sqrt{1-2 \varepsilon}
$$

die Wurzeln der quadratischen Gleichung

$$
z^{2}-2 z+2 \varepsilon=0
$$

und es ist daher $x^{2 n}+y^{2 n}$ der Coefficient von $u^{2 n}$ in der Entwickelung des Ausdrucks

$$
\frac{1}{1-u x}+\frac{1}{1-u y}=\frac{2(1-u)}{1-2 u+2 \varepsilon u^{2}}
$$

mit Rücksicht hierauf endlich erscheint $\alpha_{k}$ als Entwickelungscoefficient, nämlich als Coefficient von $\varepsilon^{n-k} \cdot u^{2 n}$ in der Entwickelung von

$$
\frac{1}{2^{n}} \cdot \frac{1-u}{1-2 u+2 \varepsilon u^{2}} \text {. }
$$

Entwickelt man diesen Ausdruck zunächst nach Potenzen von $\varepsilon$, so erhält 
man als Coefficienten von $\varepsilon^{n-k}$ :

$$
\frac{(-1)^{n-k}}{2^{n}} \cdot \frac{(1-u) \cdot\left(2 u^{2}\right)^{n-k}}{(1-2 u)^{n-k+1}}=\frac{(-1)^{n-k}}{2^{k}} \cdot \frac{(1-u) u^{2(n-k)}}{(1-2 u)^{n-k+1}}
$$

und indem man dies wieder nach $u$ entwickelt, findet sich der gesuchte Coefficient von $u^{2 n}$ :

$$
\begin{aligned}
\alpha_{k} & =\frac{(-1)^{n-k}}{2^{k}}\left\{\frac{n-k+1 \cdot n-k+2 \ldots n+k}{1.2 \ldots 2 k} \cdot 2^{2 k}-\frac{n-k+1 . n-k+2 \ldots n+k-1}{1.2 \ldots 2 k-1} \cdot 2^{2 k-1}\right\} \\
& =(-1)^{n-k} \cdot \frac{n}{k} \cdot \frac{n-k+1 . n-k+2 \ldots n+k-1}{1.2 \ldots 2 k-1} \cdot 2^{k-1} .
\end{aligned}
$$

Dieser Formel entzieht sich nur der Coefficient des ersten Gliedes; indem man $k=0$ setzt, erhält man als Coefficient von $u^{2 n}$ in der Entwickelung des Ausdrucks

$$
\frac{(-1)^{n}}{2^{n}} \cdot \frac{(1-u) \cdot\left(2 u^{2}\right)^{n}}{(1-2 u)^{n+1}}
$$

nach aufsteigenden Potenzen von $u$ offenbar:

$$
\alpha=(-1)^{n} \text {. }
$$

Die Formel für $\alpha_{k}$ giebt noch:

$$
\frac{\alpha_{k}}{2^{k} \cdot(n . n-1 \ldots n-k+1)^{2}}=\frac{(-1)^{n-k}}{1.2 \ldots 2 k} \cdot \frac{n . n+1 . n+2 \ldots n+k-1}{n \cdot n-1 . n-2 \ldots n-k+1}
$$

und so wird endlich die gesuchte Eliminationsgleichung, wenn man in (15.) diese Coefficienten einführt und den gemeinsamen Factor $(-1)^{n}$ übergeht:

$$
\begin{aligned}
& 0=U^{2}-\frac{\varrho^{2}}{1.2} \cdot \frac{n}{n} A^{2}\left(U^{2}\right)+\frac{\varrho^{4}}{1.2 .3 .4} \cdot \frac{n . n+1}{n . n-1} \Delta^{4}\left(U^{2}\right) \\
&-\frac{\varrho^{6}}{1.2 .3 .4 .5 .6} \cdot \frac{n . n+1 . n+2}{n \cdot n-1 . n-2} \Delta^{6}\left(U^{2}\right)+\cdots
\end{aligned}
$$

Mit geringer Modification der Ausdrucksweise kann man den angegebenen Weg die Function $\Omega$ darzustellen, nun folgendermassen zusammenfassen:

Ist $\Omega$ eine homogene Function $n^{\text {ter }}$ Ordnung, welche die Gleichung $\Delta^{2} \Omega=0$ befriedigt, also als das Product einer Kugelfunction $n^{\text {ter }}$ Ordnung mit der $n^{\text {ten }}$ Potenz des Radius dästellbar ist, so ist der Ausdruck

$$
\Omega^{2}-\frac{n}{n} \cdot \frac{r^{2} \Delta^{2}\left(\Omega^{2}\right)}{1.2}+\frac{n . n+1}{n . n-1} \cdot \frac{r^{4} \Delta^{4}\left(\Omega^{2}\right)}{1.2 .3 .4}-\cdots
$$

stets in $n$ lineare Factoren zerlegbar. Verbindet man diese paarweise, sind zwei so zusammengehörige

$$
\alpha x+\beta y+\gamma z, \quad \alpha^{\prime} x+\beta^{\prime} y+\gamma^{\prime} z,
$$


und bezeichnet man dann das Product der $n$ Ausdrücke

durch $V$, so ist immer

$$
\left|\begin{array}{lll}
x & y & z \\
\alpha & \beta & \gamma \\
\alpha^{\prime} & \beta^{\prime} & \gamma^{\prime}
\end{array}\right|
$$

$$
\text { (17.) } \Omega=C\left\{V-\frac{r^{2} \Delta^{2} V}{2.2 n-1}+\frac{r^{4} \Delta^{4} V}{2.4 .2 n-1.2 n-3}-\cdots\right\},
$$

ioo C eine Constante bezeichnet.

Ich bemerke noch, dass dies Product $V$ zwar auf $1.3 .5 \ldots 2 n-1$ verschiedene Arten durch verschiedene Combination der linearen Factoren gebildet werden kann; aber nur auf eine einzige Weise, wenn diese Factoren sämmtlich reell sein sollen. Denn die linearen Factoren des Ausdrucks (16.), welche die Schnittpunkte von $V=0 \mathrm{mit}$

$$
x^{2}+y^{2}+z^{2}=0
$$

darstellen, sind offenbar sämmtlich imaginär; man kann also reelle Factoren in $V$ nur dadurch erzielen, dass man zu deren Bildung je zwei conjugirte Factoren des Ausdrucks (16.) verbindet.

Die Form von $\Omega$, in welcher $V$ ein Product linearer Ausdrücke ist, steht mit denjenigen Kugelfunctionen im genauesten Zusammenhange, welche aus der Entwickelung der reciproken Quadratwurzel der Entfernung zweier Punkte hervorgehen. Eine solche Function, multiplicirt mit den $n^{\text {ten }}$ Potenzen der Radien, entsteht, wenn $V$ die $n^{\text {te }}$ Potenz eines linearen Ausdrucks wird. Bezeichnen wir das Resultat durch $V_{n}$, so dass

$$
\begin{aligned}
V_{n}=\left(x x_{0}+y y_{0}+z z_{0}\right)^{n}- & \frac{r^{2} r_{0}^{2}}{2.2 n-1}\left(x x_{0}+y y_{0}+z z_{0}\right)^{n-2} \\
& +\frac{r^{4} r_{0}^{4}}{2.4 .2 n-1.2 n-3}\left(x x_{0}+y y_{0}+z z_{0}\right)^{n-4}-+\cdots,
\end{aligned}
$$

so kann man die allgemeinste Function $n^{\text {ter }}$ Ordnung $\Omega$ in der durch (17.) dargestellten Form sich dadurch entstanden denken, dass $x$ mal hintereinander das Differential von $V_{n}$ in Bezug auf $x_{0}, y_{0}, z_{0}$ gebildet ist, und statt der Incremente beliebige Constante, und zwar jedesmal andere, substituirt wurden.

Carlsruhe, im Juni 1861. 